\title{
Automatic seizure detection based on the combination of newborn multi-channel EEG and HRV information
}

\author{
Mostefa Mesbah ${ }^{1 *}$, Malarvili Balakrishnan ${ }^{2}$, Paul B Colditz ${ }^{3}$ and Boualem Boashash ${ }^{3,4}$
}

\begin{abstract}
This article proposes a new method for newborn seizure detection that uses information extracted from both multi-channel electroencephalogram (EEG) and a single channel electrocardiogram (ECG). The aim of the study is to assess whether additional information extracted from ECG can improve the performance of seizure detectors based solely on EEG. Two different approaches were used to combine this extracted information. The first approach, known as feature fusion, involves combining features extracted from EEG and heart rate variability (HRV) into a single feature vector prior to feeding it to a classifier. The second approach, called classifier or decision fusion, is achieved by combining the independent decisions of the EEG and the HRV-based classifiers. Tested on recordings obtained from eight newborns with identified EEG seizures, the proposed neonatal seizure detection algorithms achieved $\mathbf{9 5 . 2 0 \%}$ sensitivity and $88.60 \%$ specificity for the feature fusion case and $95.20 \%$ sensitivity and $94.30 \%$ specificity for the classifier fusion case. These results are considerably better than those involving classifiers using EEG only $(80.90 \%, 86.50 \%)$ or HRV only $(85.70 \%, 84.60 \%)$.
\end{abstract}

Keywords: Time-frequency representation, Heart rate variability, EEG, Newborn seizure, Seizure detection, Features fusion, Classifier combination, TFD, MBD, IF

\section{Introduction}

Seizures occur when a large number of cortical neurons undergo a sudden, excessive, and synchronized depolarization. The reported incidence of newborn seizures varies enormously from 0.15 to $15 \%$, depending on the population studied [1]. Newborn seizures have been associated with increased rates of long-term chronic neurological morbidity and neonatal mortality [2]. Hypoxic ischemic encephalopathy, intracranial hemorrhage, infarcts or neonatal strokes, intracranial infection, and biochemical imbalances within the CNS constitute about $90 \%$ of the etiologies of neonatal seizures [3]. Early detection of seizure in the newborn is, therefore, crucial as prolonged untreated seizures can result in long-term neurological damage.

Newborn seizure manifestations can be classified as clinical or electrical (electroencephalogram-EEG). The clinical signs involve stereotypical physical behaviors

\footnotetext{
* Correspondence: mmesbah@ieee.org

${ }^{1}$ School of Mechanical and Chemical Engineering, The University of Western Australia, Crawley, WA 6009, Australia

Full list of author information is available at the end of the article
}

such as sustained eye opening with ocular fixation, repetitive blinking, or fluttering of the eyelids, drooling, sucking, and other slight facial manifestations [4]. Unlike in adults and children, these clinical manifestations are usually subtle in newborns and, therefore, require a constant attention of the medical staff to be detected. As a result, growing attention has been focused toward developing computerized methods to automate the newborn EEG seizure detection process [5-14].

Newborn seizures manifest themselves in the EEG as repetitive waveforms that are distinctly different from the normal random-like background cerebral activity. These characteristics have been exploited by a number of researchers when designing automated seizures detection methods. Some of these methods are based on quantifying this periodicity in (1) the time domain using correlation function [5,15], changes in model structure $[10,16,17]$, synchronization between channels [18], and wave-sequence analysis [3,19], (2) the frequency domain using power spectral density [11,20,21], and (3) the time-frequency domain using quadratic time-frequency 
distributions [14,22-24] and atomic decomposition $[25,26]$. Faul et al. [27] compared the detection methods proposed by Liu et al. [5], Gotman et al. [11] and Celka and Colditz [10] using a common EEG database. The authors concluded that all three methods failed to reliably identify neonatal seizures.

Other researchers adopted a pattern recognition approach to the problem of seizure detection. The approach consisted of extracting and selecting discrete features from different analysis domains and using them to train classifiers. Different classifiers have been used, namely artificial neural networks $[13,21,28,29]$, discriminant [30], and support vector machine [31]. Despite this effort, a reliable and robust automatic recognition of newborn EEG seizure remains a challenging task. This is due to a number of factors such the large inter- and intra-patient variability of the newborn EEG seizure morphology, the lack of consensus among EEG experts on what constitute an EEG seizure, and the complete reliance on the EEG as the only source for information.

Authors had realized the need to take into account other physiological data [7]. In particular [32-34], the present authors showed that changes in heart rate variability (HRV) generally accompany electrical manifestation of newborn seizures. These findings suggest that HRV provides important information that can be used in seizure detection. However, apart from few attempts such as [32-36], there has been little work done using physiological signals, other than EEG, for automatic identification of seizure in newborns. Therefore, in this article, we propose new methods for newborn EEG seizure detection by exploiting information extracted from both HRV and multi-channel EEG to improve the accuracy and robustness of the detection process, as previously suggested in $[7,37]$. The success of this endeavor would result in a decrease on complete reliance on the
EEG as the sole information source for detection of newborn seizure and, therefore, a reduction of false detections caused by confounding factors in EEG. Although it uses similar structures as the method proposed in [38], the present approach differs from [38] in a significant number of points, such as the nature of feature extracted, the use of feature selection, and the rules for combining the classifiers as detailed within the text.

In this article, we investigate the idea and compare the performance of fusing the information extracted from EEG and HRV for newborn seizure detection. This fusion is performed at two different levels, namely feature level and decision level. In the feature fusion approach, features derived from EEG and HRV are combined into a single feature vector before feeding it to a single classifier. In the decision fusion, two classifiers are used; one based on features extracted from EEG and the other from features obtained from HRV. The final decision is obtained by integrating the separate decisions of the two classifiers. We also compare our detectors to the ones proposed by Greene et al. [38] and discuss the possible sources of the differences in performance. We end the article by discussing different limitations of this study and how to overcome them in the future.

This article is organized as follows. "Materials and methods" section addresses the different components involved in the development of the process for newborn seizure detection. The performances of the proposed detectors are evaluated and the results are discussed in "Data acquisition" section. In the final section, conclusions about this study are presented.

\section{Materials and methods}

In this article, two approaches for combining information extracted from multi-channel EEG and singlechannel HRV are investigated. Figures 1 and 2 show the

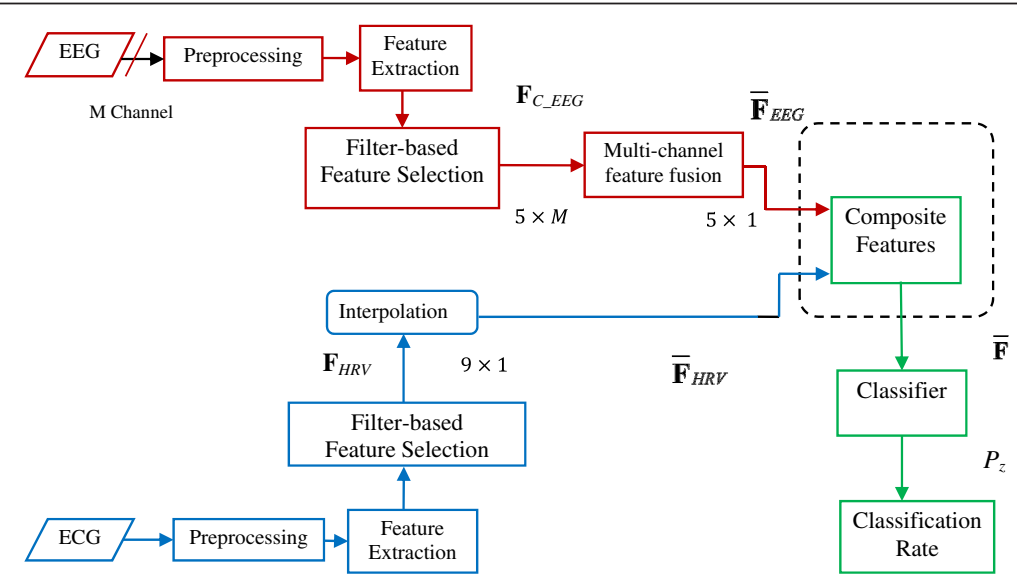

Figure 1 Newborn seizure detection process based on EEG-HRV feature fusion. The optimal feature of EEG, $\bar{F}_{E E G}$, and HRV, $\bar{F}_{H R V}$, are combined as $\bar{F}$ before fed into classifier to obtain classification rate, $P Z$. 


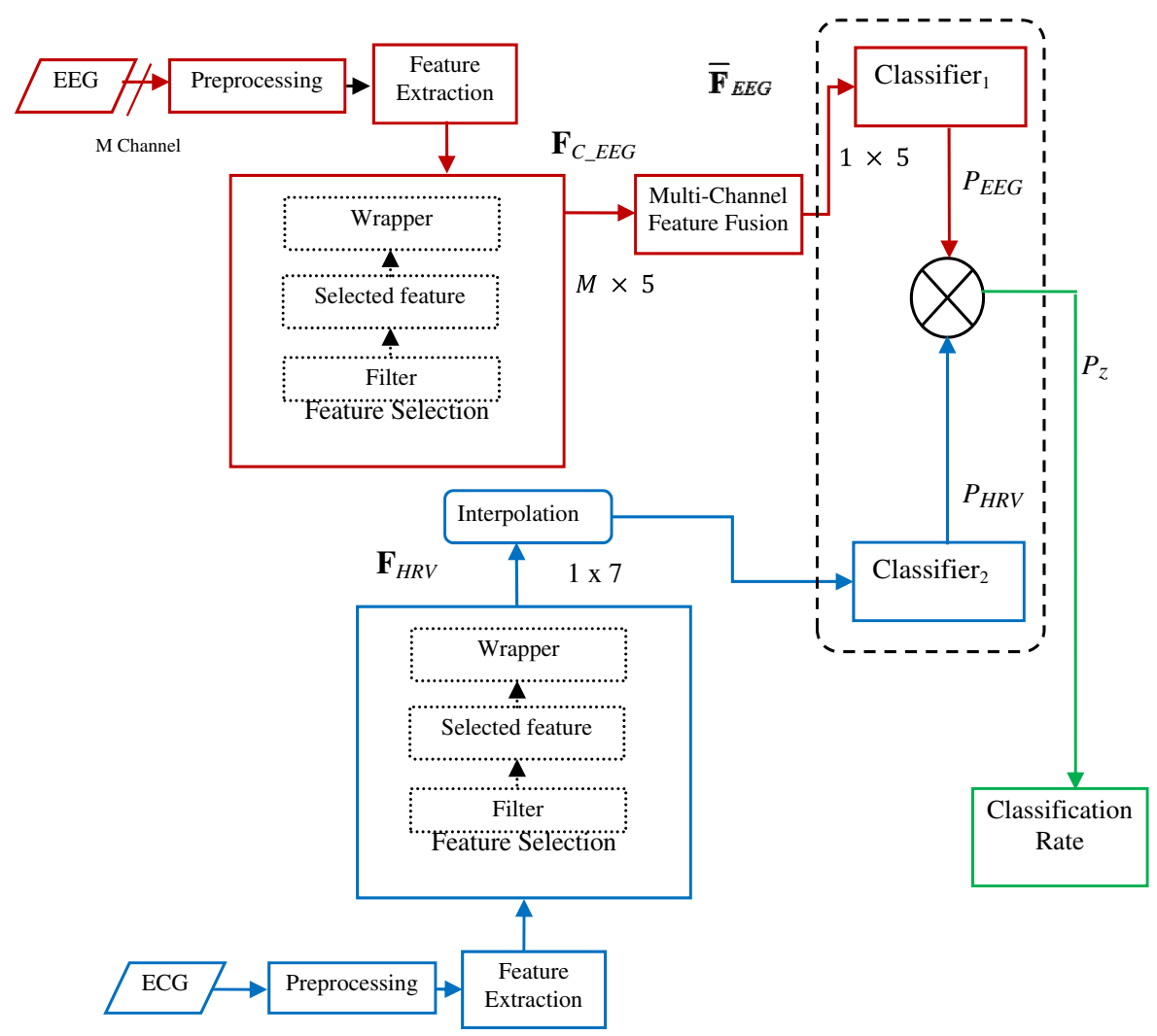

Figure 2 Flow chart for newborn seizure detection based on EEG-HRV classifier/decision fusion. The optimal feature of EEG, $\bar{F}_{E E G}$, and HRV, $\bar{F}_{\text {HRV }}$ are fed into separate classifiers. The independent decisions of these classifiers, $\mathrm{P}_{\mathrm{EEG}}$ \& $\mathrm{P}_{\mathrm{HRV}}$, were then, combined to produce an overall classification rate $P z$.

different components of the two newborn seizure detection approaches. Both share the following processing steps: preprocessing, feature extraction, feature selection, and fusion. Each of these steps is detailed below.

\section{Data acquisition}

The eight EEG-electrocardiogram (ECG) records used in this study were acquired from eight full-term newborn babies (GA: 40-42 weeks) admitted to the Royal Brisbane Hospital, Brisbane, Australia. Twenty EEG channels and one ECG channel were simultaneously recorded using Medelec Profile System (Oxford Instruments, UK). The EEG channels were obtained from 14 electrodes, placed according to the international 10-20 standard, using longitudinal bipolar montage (see Table 1). The EEG seizures were identified and annotated by a pediatric neurologist from Royal Children Hospital, Brisbane, Australia. The eight EEG recordings totaling $2.8 \mathrm{~h}$ (mean $21.22 \mathrm{~min}$ ) contained 13 partial seizure events of a total duration of 33 min (mean 2.54 min). The EEG and ECG were filtered using an analog band-pass filter with cut-off frequencies 0.5 and $70 \mathrm{~Hz}$ prior to being digitized at a rate of $256 \mathrm{~Hz}$. A $50-\mathrm{Hz}$ notch filter was then used to remove the effects of power line interferences. As the aim of this article is to combine the information from EEG and ECG data, only EEG recordings or parts of the recordings accompanied with ECG have been selected. Also, sections of the recordings exhibiting large movement artifacts, saturation, or those associated with detached electrodes have been eliminated from further processing.

Table 1 Bipolar montage used to acquire the EEG data

\begin{tabular}{|c|c|}
\hline Right hemisphere & Left hemisphere \\
\hline 1 (F4-T4) & 4 (F3-T3) \\
\hline 2 (T4-T6) & 5 (T3-T5) \\
\hline $3(\mathrm{T6}-\mathrm{O} 2)$ & $6(\mathrm{~T} 5-\mathrm{O} 1)$ \\
\hline 7 (F4-C4) & 10 (F3-C3) \\
\hline 8 (C4-P4) & 11 (C3-P3) \\
\hline 9 (P4-O2) & 12 (P3-O1) \\
\hline $13(\mathrm{~T} 4-\mathrm{C} 4)$ & $15(\mathrm{Cz}-\mathrm{C} 3)$ \\
\hline $14(\mathrm{C} 4-\mathrm{Cz})$ & 16 (C3-T3) \\
\hline 17 (T6-P4) & 19 (Pz-P3) \\
\hline 18 (P4-Pz) & 20 (P3-P5) \\
\hline
\end{tabular}




\section{Preprocessing}

\section{Preprocessing ECG}

To conform with the minimum requirement of the Task Force of the European Society of Cardiology and the North American Society of Pacing and Electrophysiology [39], we divided the ECG into 64-s segments (epochs). In this study, we randomly selected 21 seizure-related and 13 non-seizure-related non-overlapping ECG segments collected from the eight recordings. In a first step, the raw ECG was filtered using a 60th-order band-pass finite impulse response filter with cutoff frequencies of 8 and 18 Hz. This was done to allow frequencies associated with the QRS waveforms to pass while stopping artifacts and non-QRS waveforms such as the $\mathrm{P}$ and $\mathrm{T}$ waves. A reliable QRS detection algorithm was used to locate the $\mathrm{R}$ points in the ECG [33]. Errors in the R point detection were corrected using timing analysis. The $R R$ interval time series was obtained by taking the time difference between consecutive $\mathrm{R}$ points. The instantaneous heart rate (IHR) was then computed as the inverse of the RR interval. The IHR time series was transformed into an evenly time-sampled one using cubic spline interpolation followed by resampling at $4 \mathrm{~Hz}$ and detrending. The resulting signal constituted the HRV used in this study. More details can be found in [33,34].

\section{Preprocessing EEG}

The multi-channel EEG was filtered using a low-pass filter with a cutoff frequency of $8 \mathrm{~Hz}$ and resampled at 20 Hz. This choice has been done for different reasons: (1) It has been shown that more than $95 \%$ of spectral energy in the newborn EEG is concentrated in the delta and theta frequency bands $(0.4-8 \mathrm{~Hz})$ [40], (2) selecting this sampling rate significantly reduces the computational burden, and (3) this filters out high-frequency noise and artifacts such as EMG. For the sake of synchronization with the HRV, the EEG from the different channels was segmented into non-overlapping 64-s EEG epochs. These epochs were further divided into five nonoverlapping windows of $12.8 \mathrm{~s}$ each. The reason for using shorter EEG windows is that many researchers consider that the minimum acceptable duration for an EEG seizure to be around $10 \mathrm{~s}$ [11]. The synchronization of HRV and EEG epochs was needed to achieve the fusion between the two signals as illustrated in Figure 3.

\section{Feature extraction HRV features}

A total of 96 features were extracted from the time and the TF domains for each HRV epoch. A brief description of the extracted features is given below. More details can be found in [33].
Time domain features The mean, standard deviation, and Hjorth parameters (which describe the signal characteristics in terms of activity, mobility, and complexity) were computed.

Time-Frequency features As HRV is a non-stationary signal, we decided to extract features from the time-frequency domain to account for this. This process was not as straightforward as in the case of the time domain features. The time-frequency (TF) representation was obtained using the Modified-B distribution (MBD) with its parameter $\beta$ set to 0.01 [41]. The MBD has been chosen to represent the HRV in the TF domain as it was previously found to realize the best compromise in terms of cross-term reduction and TF resolution among a number of quadratic time-frequency distributions (TFDs) [34]. The MBD of a real signal, $s(t)$, is given by [41]

$$
\begin{aligned}
p(t, f)= & \iiint_{-\infty} g(v, \tau) z(u+\tau / 2) \bar{z}(u-\tau / 2) \\
& \times e^{j 2 \pi(v t-v u-f \tau)} d v d u d \tau^{\infty}
\end{aligned}
$$

where $z(t)$ is the analytic associate of $s(t)$ and $\bar{z}(t)$ its complex conjugate. The function $g(v, \tau)$ is a kernel defined in the Doppler-lag $(v, \tau)$ domain as [41]

$$
g(\nu, \tau)=\frac{|\Gamma(\beta+j \pi v)|^{2}}{|\Gamma(\beta)|^{2}}
$$

where $\Gamma$ stands for the complex-valued gamma function, $\beta$ is a real positive number that controls the trade-off between TF resolution and cross-terms suppression [41], and $|$.$| is the absolute function operator.$

Figure 4a,b represents the MBD of the HRV associated with non-seizure and seizure epochs, respectively. Accurate low-frequency (LF), mid-frequency (MF), and highfrequency (HF) components of HRV were extracted using a recently proposed TF-based multicomponent IF estimation technique [42]. The TFD of the HRV represents its energy distribution in the joint TF domain where the most valuable information is encoded in the instantaneous frequency (IF) and instantaneous amplitude (IA) of the different components. The IF shows the TF region where the signal energy is concentrated while the IA is the amplitude envelope of each TF component; that is the magnitude of the peak of the TF component as a function of time. The spread of each TF HRV component away from the IF is measured by means of the instantaneous bandwidth (IB). The article [43] provides an in-depth review and visual illustration of the concepts of IF, IA, and IB. Their computational aspects are covered in [44]. Here, the IB refers to the bandwidth of instantaneous spectrum at every time instant when the energy density of the respective component drops by $50 \%$ from its maximum. 


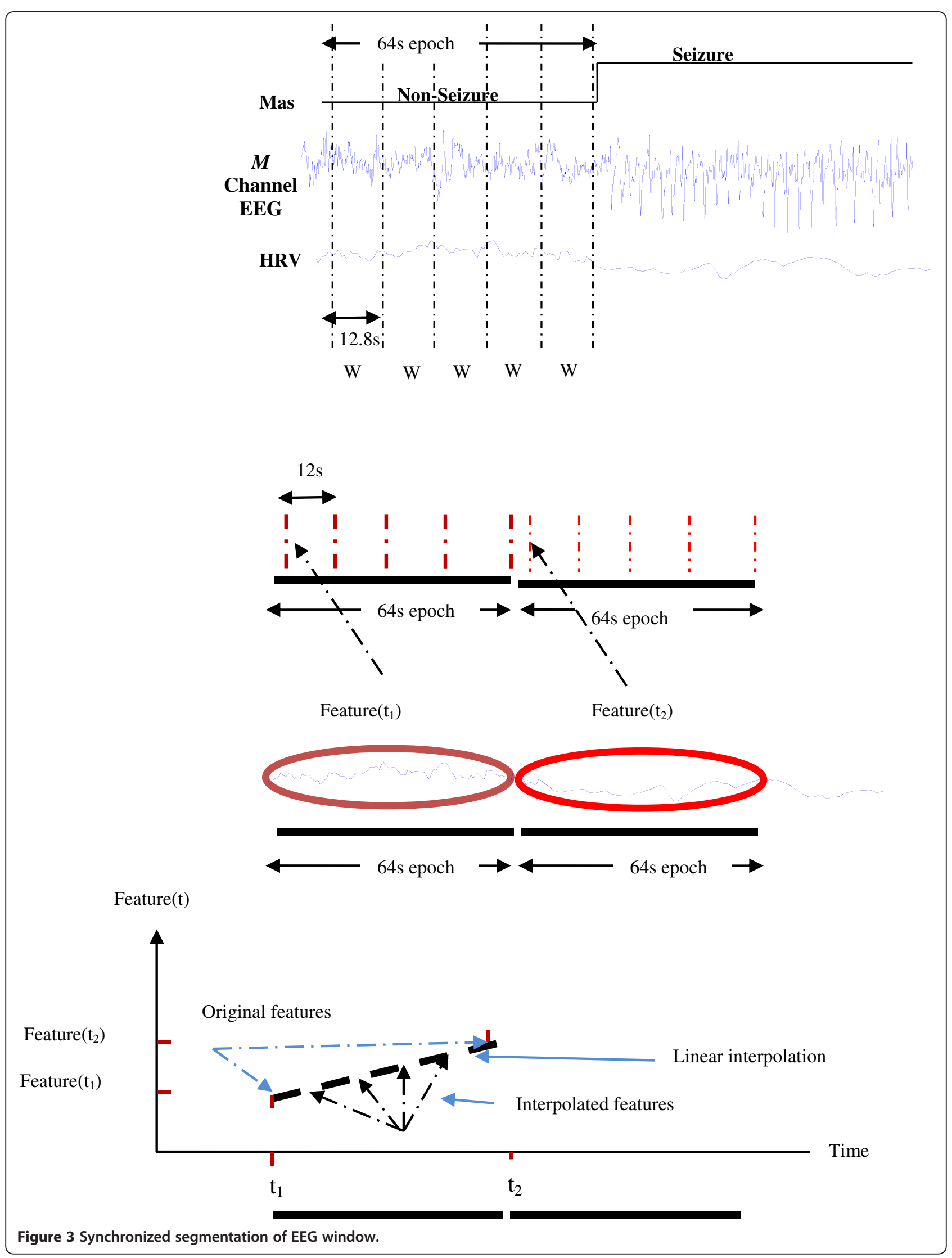


a)

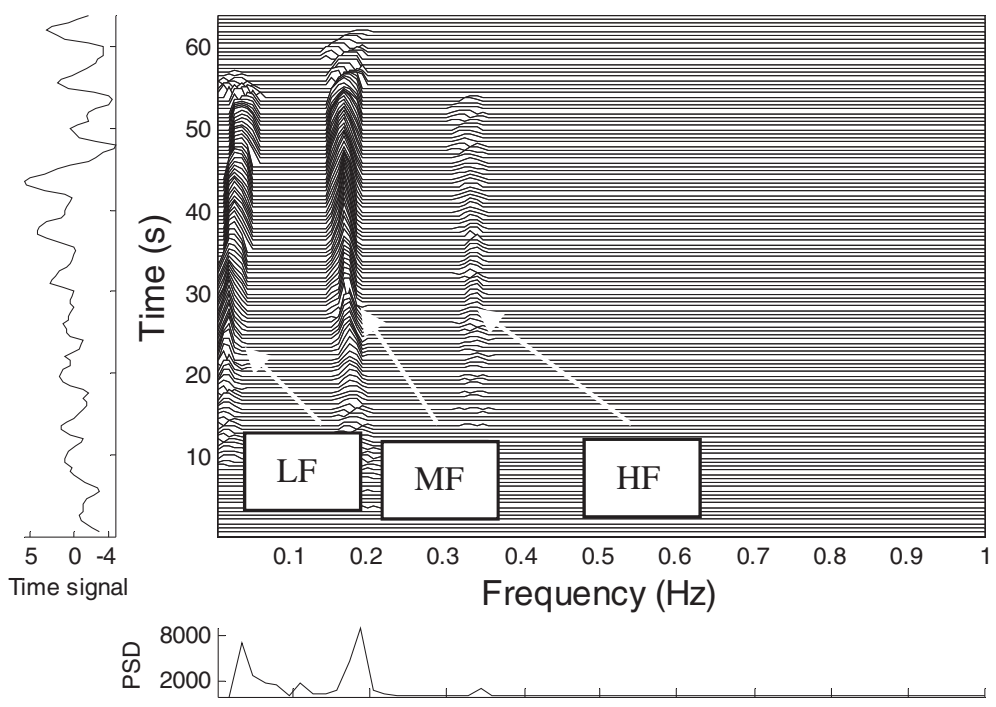

b)

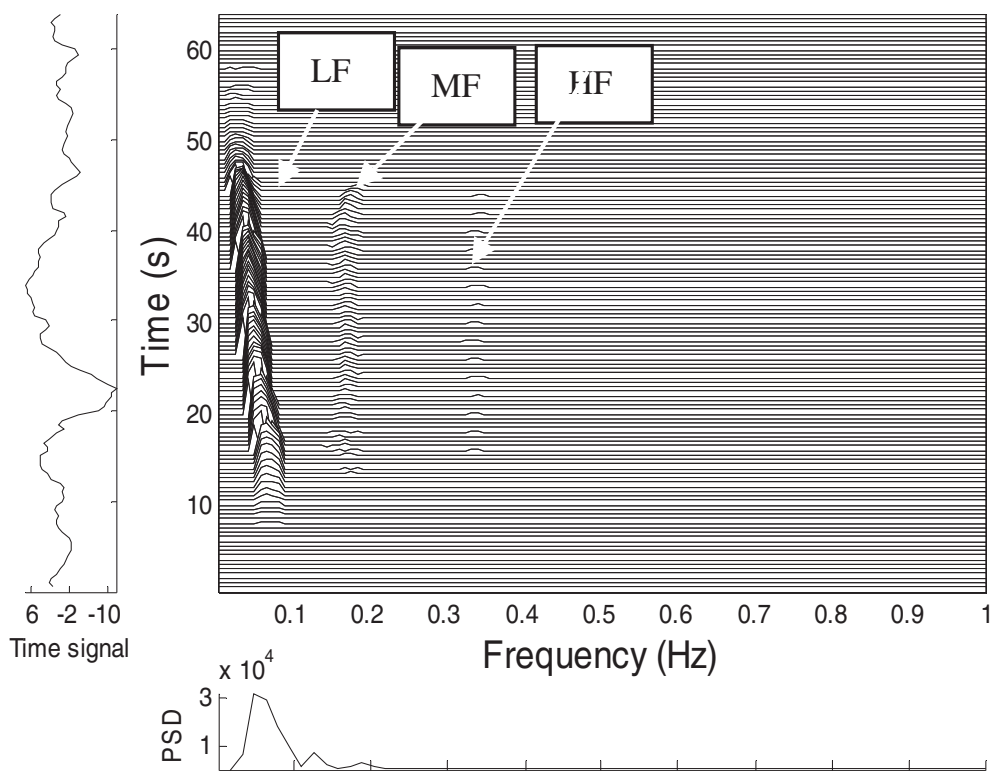

Figure 4 The modified B distribution of HRV and the components, i.e., LF, MF, and HF related to (a) non-seizure and (b) seizure epoch. The energy in LF is significantly greater compared to MF and HF that makes the components unnoticeable (see the power spectral density at the bottom of the TF plot). These figures were produced using a TFSA package [41]; a toobox for MATLAB ${ }^{\circledR}$ ).

The HRV TF features comprised:

i. Statistical quantities extracted from the IF, IB, and IA of the LF, MF, and HF components: mean, standard deviation, median, RMS, min, max, coefficient of variation, skewness, and kurtosis.

ii. The energy in LF, MF, and HF components, the total energy in all the HRV components, and the ratio of energy in the LF to HF (LF/HF). iii. The generalized Shannon entropy of the normalized TFD [33].

\section{EEG features}

A number of EEG features were extracted from each EEG segment of each EEG channel. Some of these features have previously been used by different authors in the context of seizure detection. Our aim here is to show 
that using information from different physiological sources results in better performing detectors than those based on information extracted from single sources. Some of the features have been modified to best suit our current approach. The features were extracted from time, frequency, time-frequency, and time-scale domains.

Time domain features These features include the mean, standard deviation, skewness, kurtosis, coefficient of variation, RMS and zero-crossings, and the Hjorth parameters [45]. The total nonlinear score, proposed by Liu et al. [5] as a measure of periodicity, was also included.

Frequency domain features The frequency domain representation was achieved using an FFT with Hamming window of length 218 points. The frequency domain features adopted here were similar to the one proposed by Gotman et al. [11]. They include the peak frequency, the maximum frequency, the bandwidth, and the spectral power of the dominant spectral peak of the EEG windows.

Time-scale features Discrete wavelet transform has been used in [13] for EEG seizure detection in newborns. Daubechies 4 wavelet has been used in decomposing the EEG segment into nine scales. We have extracted the optimal features identified in [13]. The features are the variance of $c d 2, c d 3, c d 4, c d 6, c d 7, c a 9$, and mean of $d 6, d 5, d 9$, where $c d i$ and $d i$ refer to the detail coefficient and detail component of scales, $i$, respectively.

Time-frequency features The TF representation was obtained using the MBD with the parameter $\beta$ set at 0.02. As in the HRV case, the MBD has been shown to realize a good compromise between cross-term reduction and TF resolution. The TF features extracted comprised the following: total TF energy, the largest and smallest singular values of the TFD and number of TF components with prefixed minimum duration. The TF components were extracted using the same technique [42] used to extract the HRV components discussed above.

For ease of comparison, all the extracted features were normalized to have zero mean and unit standard deviation. To remove redundant features and/or those with poor discriminatory power, a feature selection process was used. This process is described below.

\section{Feature selection}

Feature selection was used to select a subset from the original extracted feature set in order to avoid performance degradation and high-computational complexity [46]. This smaller set included features that were both relevant and non-redundant.
Most existing feature selection approaches belong to two categories: wrappers and filters [47]. Wrappers are techniques that include the classifier as an essential component. The classifier performance is used in the evaluation of the optimality of the selected features. The filters, on the other hand, are classifier-independent. For a pre-selected classifier, wrappers tend to give superior performance as they select features optimally adapted to the classifier. This, however, comes with a higher computational cost along with the poor generalization to other classifiers. Filters, on the other hand, are computationally efficient but since they are classifier-independent, they tend to select sub-optimal features [47].

In this article, we used feature selection methods we previously developed $[33,48,49]$ to select the optimal feature subset with minimum redundancy and maximum class discriminability. The feature selection process was considered successful if the dimensionality of the feature set was reduced while the accuracy of the classification was either improved or remained unchanged relative to the full set.

\section{Feature fusion case}

In this approach, as illustrated in Figure 1, the optimal HRV-EEG feature subsets were selected using the filterbased selection method described in [48] where it has been shown to be able to significantly reduce the number of features while maintaining a high classification performance. The optimal HRV and EEG feature selected as a result of employing the filter-based feature selection method are given below.

Selected HRV features The following nine features were selected: energy in LF, mean of IA for HF, minimum of IA for MF, TF-based Shannon entropy, STD of IA for MF, ratio of LF/HF, sum of IA for LF, sum of IA for HF (TF features), and STD of HRV (time-domain features).

Selected EEG features Five features were retained: TF energy, number of TF components (TF features), peak frequency, bandwidth of the peak frequency (frequencydomain features), and total score from Liu's technique (time-domain feature).

\section{Decision fusion case}

In this approach, shown in Figure 2, the extracted HRV and EEG features were fed to a two-phase filterwrapper-based algorithm described in [49]. The rationale for using here a filter-wrapper feature selection, instead of a filter-based one, is that the wrapper stage provides both the set of optimal features and the related best performing classifier. The selected classifier is then used in the decision fusion process. 
The filter-wrapper-based feature selection method in [49] is a two-phase process that has been used to reduce the computation load and the complexity of the search operations associated with the wrapper approach. The first phase, which acts as pre-processing phase, involves the filter [48] discussed earlier. As the result, a set of relevant features, f, with minimum redundancy and maximum class discriminability is obtained. In the second phase, the feature subset, $\mathbf{f}$, is presented as an input to the wrapper. The wrapper uses the performance of the classifier, such as the probability of error, to assess the goodness of the selected features [49]. The wrapper was evaluated using three different statistical classifiers, namely linear, quadratic, and $k$-nearest neighbor. Optimal EEG and HRV feature subsets together with the best performing classifiers were selected as a result of employing the filter-wrapper-based feature selection method.

Optimal HRV features and classifier A combination of seven HRV features, $F_{\mathrm{HRV}}$, and the $1-\mathrm{NN}$ classifier gave the best classification performance. The selected features were: energy in LF, mean of IA for HF, min of IA for MF, TF-based Shannon entropy, standard deviations of IA for MF, LF/HF ratio, sum of IA for LF, sum of IA for HF (all TF features).

Optimal EEG features and classifier A subset of five features combined with a linear classifier gave the best performance. The five features selected here were the same ones obtained using the filter-based algorithm mentioned above, namely TF energy, number of TF components (TF features), peak frequency, bandwidth of the peak frequency (frequency-domain features), and total score from Liu's technique (time-domain feature).

It is important to note that most of the features retained by the feature selection process, in both types for information fusion, are time-frequency ones. This attests to the suitability of the TF domain in representing non-stationary physiological signals such as EEG and HRV.

\section{EEG and HRV information fusion}

In order to make the EEG-HRV feature fusion possible, the frame rates of the two signals must match. In Figure 3, the EEG frame rate is five times that of the HRV and as such, there is a mismatch between the features of HRV and EEG. To deal with this issue, we investigated three different solutions: (1) assign a constant value to all 12.8-s HRV windows, (2) use linear interpolation, and (3) use higher-order polynomials. The linear interpolation was adopted as it realized a good tradeoff between performance and complexity and resulted in a smooth transition between feature values.

\section{The feature fusion approach}

As illustrated in Figure 1, the EEG epochs were preprocessed before being fed to the feature extraction and selection units. As mentioned above, from the larger set of features extracted, only five were selected. For each 12.8-s window, a $5 \times M$ matrix of features, $F_{C_{\mathrm{EEG}}}$, was obtained by concatenating the $M 5 \times 1$ feature vectors corresponding to the $M$ different channels. The matrix $F_{C_{\mathrm{EEG}}}$ was then reduced to a $5 \times 1$ vector $\bar{F}_{\mathrm{EEG}}$ by using symmetrical uncertainty-based dimension reduction method. This procedure has been shown to perform well in multi-channel EEG seizure detection [50]. In terms of features, this is equivalent to representing the $M$ channels by a single representative channel prior to feature fusion.

As explained earlier, for the case of HRV, a total of 96 features was extracted from the 64-s epoch, and an optimal 9 features represented by the $9 \times 1$ vector $\bar{F}_{\mathrm{HRV}}$ were used to train the classifiers. To ensure matching segmentation between EEG and HRV features, HRV features were linearly interpolated (as shown in Figure 3). The resulting feature vectors $\bar{F}_{\text {HRV }}$ and $\bar{F}_{\text {EEG }}$ were concatenated to form a single composite feature vector, $\bar{F}$, given by

$$
\bar{F}=\left[\begin{array}{l}
\bar{F}_{\mathrm{EEG}} \\
\bar{F}_{\mathrm{HRV}}
\end{array}\right]
$$

The composite feature vector was then fed to a number of statistical classifiers. The classifiers used in our investigation were linear, quadratic, and $k-\mathrm{NN}$ with $k=1$, 3 , and 5 using a leave-one-out cross validation. It has been shown that leave-one-out cross validation is suitable in the small data case [51].

\section{The classifier/decision fusion approach}

In the decision fusion approach, two separate classifiers were used for EEG and HRV as shown in Figure 2. The independent decisions of these classifiers were, then, combined to produce an overall decision. The multichannel EEG and the HRV were processed as in the feature fusion scheme previously described prior to being combined at the decision level. In the current approach, however, the extracted HRV and EEG features were submitted to a two-phase wrapper-based feature selection process described in [48]. The matched feature vectors associated with the HRV and EEG where then fed to the two separate classifiers previously identified as optimum, namely linear and 1-NN classifiers. The independent decisions from the classifiers were finally fused using the combination rules described in [52] for an overall classification into seizure and non-seizure activities. The tested combining rules were mean, max, min, product, 


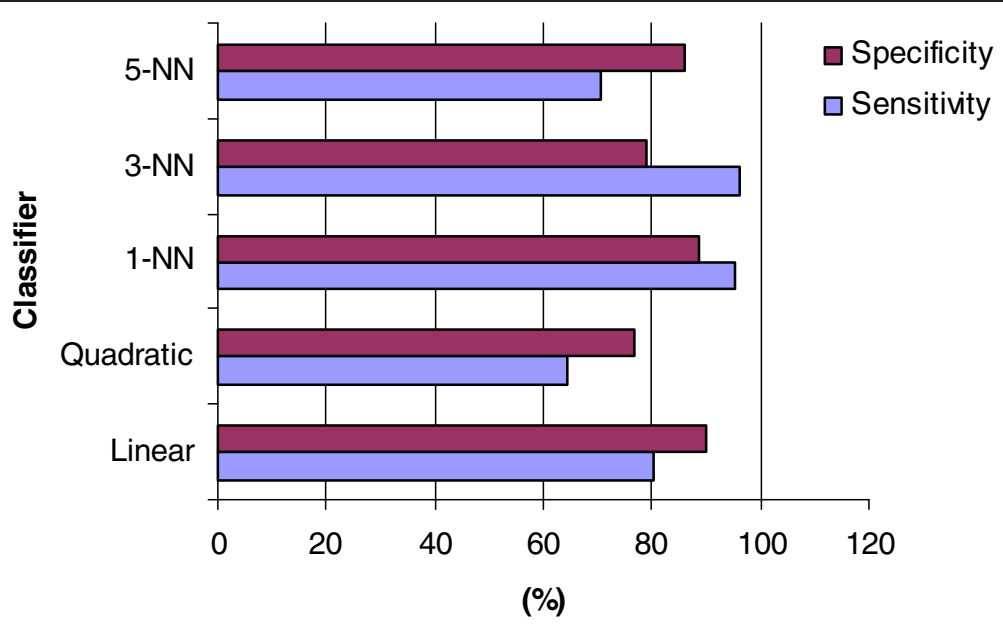

Figure 5 Performance of different classifier for seizure detection based on newborn EEG and HRV feature fusion.

sum, and majority vote rules. The results are presented in the following section.

\section{Performance analysis and discussion}

\section{Seizure detection performance based on feature fusion}

We first compared the performance of seizure detection based on feature fusion using different statistical classifiers. The performance of the EEG-HRV feature fusion scheme, as determined by the classification results in terms of sensitivity and specificity, is presented in Figure 5. Using the selected composite features discussed above, Figure 5 shows that the $1-\mathrm{NN}$, with $95.20 \%$ sensitivity (SEN) \& $88.60 \%$ specificity (SPE), achieved the best overall performance. The 3-NN achieved a SEN slightly higher than the SEN yielded by $1-\mathrm{NN}$ but at the expense of lower SPE (78\%). The performances of the other classifier investigated were relatively poorer.
Seizure detection performance-based classifier fusion Figure 6 illustrates the performance results for the classifier combination case. The mean, max, min, product, and sum rules achieved 95.20\% SEN \& 94.30\% SPE. The majority vote rules achieved 100\% SPE at the expense of low SEN (71.4\%). The mean, max, min, product, and sum rules were therefore found to give the best overall performance for this application. That most combination rules gave the same results can be explained by the fact that these simple rules were actually developed from "sum" and "product" rules as detailed in ([53], Chapter 5).

\section{Seizure detection performance: combined EEG-HRV} versus EEG only and HRV only

We assessed the value of both fusion strategies for newborn seizure detection by comparing their performances to those based on either HRV or EEG (i.e., without fusion). Table 2 shows the performances in terms of

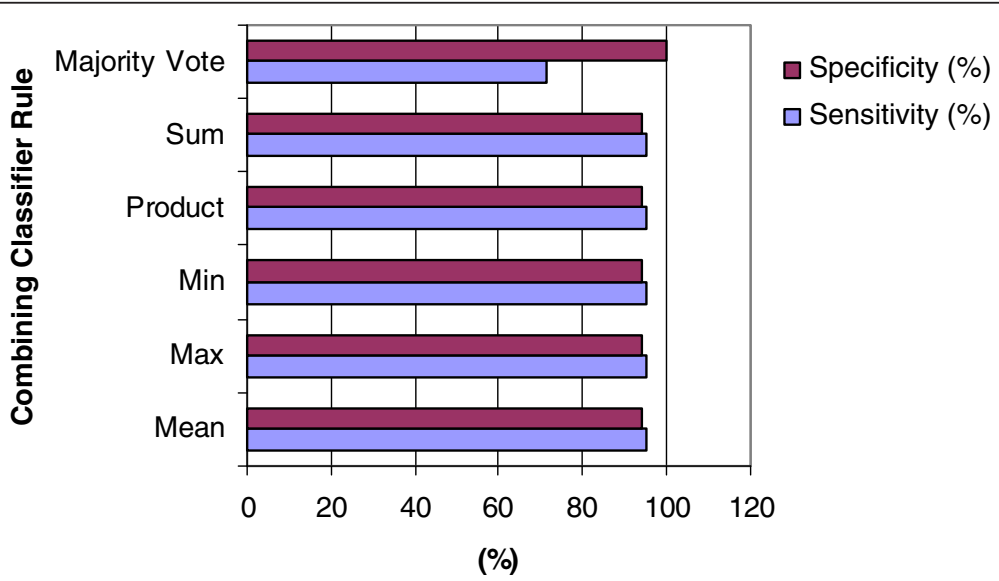

Figure 6 Performance comparison of classifier fusion of EEG and HRV using various classifier combination rules. 
Table 2 Performance comparison of newborn seizure detection using individual signal classification (EEG or HRV) and the proposed fusion configuration

\begin{tabular}{|c|c|c|c|c|c|c|c|c|}
\hline \multirow[t]{2}{*}{ Algorithm } & \multicolumn{2}{|l|}{ EEG } & \multicolumn{2}{|l|}{ HRV } & \multicolumn{2}{|c|}{ Feature fusion } & \multicolumn{2}{|c|}{ Classifier fusion } \\
\hline & SEN & SPE & SEN & SPE & SEN & SPE & SEN & SPE \\
\hline Proposed & 80.90 & 86.50 & 85.70 & 84.60 & 95.20 & 88.60 & 95.20 & 94.30 \\
\hline Liu & 60.60 & 61.50 & - & - & 70.10 & 78.60 & 85.70 & 67.50 \\
\hline Gotman & 72.10 & 69.20 & - & - & 79.00 & 76.80 & 82.60 & 86.60 \\
\hline
\end{tabular}

sensitivity and specificity. The feature-based combination classifier achieved $95.20 \%$ SEN \& $88.60 \%$ SPE compared to $80.90 \%$ SEN \& $86.50 \%$ SPE using the EEG features alone or $85.70 \%$ SEN \& $84.60 \%$ SPE using the HRV features only. This shows that the combined features have significantly improved the sensitivity of newborn seizure detection compared to the sensitivity achieved using either HRV or EEG features alone. The improvement of specificity through feature fusion, although not as dramatic as the sensitivity, was also remarkable $(+2.10 \%)$. The proposed seizure detector based on the classifier fusion accomplished 95.20\% SEN \& 94.30\% SPE compared to $80.90 \%$ SEN \& $86.90 \%$ SPE using the EEG features alone and $85.70 \%$ SEN \& $84.60 \%$ SPE using the HRV features only. Here, an even more significant overall improvement was obtained compared to the feature fusion process.

\section{Effects of the feature selection on the seizure detection performance}

To assess the benefit of the feature selection process, we compared the performance of the full feature set and the reduced feature set-based classifiers for the cases of HRV (Table 3), EEG (Table 4), and the combined EEGHRV (Table 5). Table 5 clearly shows the positive impact of the feature selection on the performance of the classification. The reduced dimension-based classifiers outclassed the full dimension based ones in both sensitivity and specificity. The good achievement of the feature selection algorithms was also visible for the cases of EEGonly and HRV-only classifiers and was accompanied, in

Table 3 Classification accuracy using original HRV features against the optimal feature subset

\begin{tabular}{|c|c|c|c|c|}
\hline \multirow[t]{2}{*}{ Classifier } & \multirow{2}{*}{$\begin{array}{l}\frac{\text { Full-set }}{\text { (96 features) }} \\
\text { Error rate }\end{array}$} & \multirow{2}{*}{$\begin{array}{c}\frac{\text { Filter }}{\text { (9 features) }} \\
\text { Error rate }\end{array}$} & \multicolumn{2}{|c|}{ Wrapper } \\
\hline & & & $\begin{array}{l}\text { Number of } \\
\text { features }\end{array}$ & $\begin{array}{l}\text { Error } \\
\text { rate }\end{array}$ \\
\hline Linear & 0.32 & 0.25 & 7 & 0.18 \\
\hline Quadratic & 0.62 & 0.36 & 6 & 0.21 \\
\hline $1-\mathrm{NN}$ & 0.39 & 0.15 & 7 & 0.15 \\
\hline 3-NN & 0.34 & 0.21 & 8 & 0.16 \\
\hline $5-\mathrm{NN}$ & 0.40 & 0.26 & 7 & 0.22 \\
\hline
\end{tabular}

all the three cases, by significant computational savings as can be seen in Table 6 .

The performance of Gotman's and Liu's algorithms with and without fusion

To further evaluate the added value resulting from combining the information extracted from EEG and HRV, we decided to investigate how Liu et al.'s [5] and Gotman et al.'s [11] algorithms would benefit by using the extra information provided by the HRV. Our proposed detectors used a set of $d$-dimensional feature vectors that characterize the EEG seizure and non-seizure patterns as input to a supervised statistical classifier. Therefore, in Liu's technique, total score is used as a feature to distinguish the EEG seizure from the non-seizure. The feature was fused with HRV features using the two proposed combination strategy. In Gotman's case, the frequency of the dominant spectral peak, bandwidth of the dominant spectral peak, and spectral power at the dominant spectral peak were extracted from each EEG window. The features were then combined with HRV features using the two proposed fusion approaches. The results of such attempts are shown in Table 2 .

Liu et al.'s algorithm achieved 60.60\% SEN \& 61.50\% SPE. Using the HRV features as complementary information at both feature and decision levels improved the sensitivity and the specificity of the seizure detection algorithm considerably (SEN: $+9.50 \% \&+25.10 \%$ and SPE: $+17.10 \%$ $\&+6.00 \%)$. Gotman et al.'s algorithm accomplished $72.10 \%$ SEN \& 69.20\% SPE. Fusing the EEG with the HRV at the decision level improved both the sensitivity and specificity

Table 4 Classification accuracy using original EEG feature set against the optimal feature subset

\begin{tabular}{|c|c|c|c|c|}
\hline \multirow[t]{2}{*}{ Classifier } & \multirow{2}{*}{$\begin{array}{l}\text { Full-set } \\
\text { Error rate }\end{array}$} & \multirow{2}{*}{$\begin{array}{c}\text { Filter } \\
\text { (5 features) } \\
\text { Error rate }\end{array}$} & \multicolumn{2}{|c|}{ Wrapper } \\
\hline & & & $\begin{array}{l}\text { Number of } \\
\text { features }\end{array}$ & Error rate \\
\hline Linear & 0.39 & 0.24 & 5 & 0.24 \\
\hline Quadratic & 0.43 & 0.37 & 4 & 0.36 \\
\hline $1-N N$ & 0.45 & 0.33 & 5 & 0.33 \\
\hline $3-N N$ & 0.53 & 0.39 & 4 & 0.37 \\
\hline 5-NN & 0.47 & 0.30 & 5 & 0.30 \\
\hline
\end{tabular}


Table 5 Classification accuracy using original combined EEG feature set against the optimal combined feature subset

\begin{tabular}{|c|c|c|c|c|c|c|c|c|}
\hline \multirow[t]{2}{*}{ Algorithm } & \multicolumn{2}{|c|}{$\begin{array}{l}\text { EEG } \\
\text { (linear classifier) }\end{array}$} & \multicolumn{2}{|c|}{$\begin{array}{l}\text { HRV } \\
\text { (1-NN classifier) }\end{array}$} & \multicolumn{2}{|c|}{$\begin{array}{l}\text { Feature fusion } \\
\text { (1-NN classifier) }\end{array}$} & \multicolumn{2}{|c|}{$\begin{array}{l}\text { Classifier fusion: } \\
\text { linear classifier \& } \\
\text { 1-NN classifier }\end{array}$} \\
\hline & SEN & SPE & SEN & SPE & SEN & SPE & SEN & SPE \\
\hline Proposed classifiers + optimal feature sets & 80.90 & 86.50 & 85.70 & 84.60 & 95.20 & 88.60 & 95.20 & 94.30 \\
\hline Proposed classifiers + full feature sets & 76.19 & 64.70 & 56.90 & 70.90 & 46.90 & 80.90 & 92.30 & 61.50 \\
\hline
\end{tabular}

the algorithm significantly (SEN: $+6.90 \%$, SPE: $+7.60 \%)$. A considerable improvement of the specificity and sensitivity was also achieved by combining the EEG and the HRV at feature level (SEN: $+10.50 \%$, SPE: $+17.40 \%)$.

From Table 2, it is evident that Liu et al.'s and Gotman et al.'s algorithms would benefit from the complementary information provided by HRV features. Based on the results of "EEG features" section, a potential explanation of the relatively poor performance of the two algorithms, compared to the proposed algorithms, has to do with the fact that EEG features sets used by the two algorithms were not good enough to achieve high discrimination between seizures and non-seizures.

\section{Detection performance comparison with Greene's algorithm}

In an attempt to combine EEG and ECG information to detect newborn seizures, Greene et al. [38] proposed two approaches, namely patient-specific and patient-independent. Both approaches were considered with fusion at feature-level (referred to as early integration or EI) and at decision-level (referred to as late integration or LI). The authors reported that the patient-specific approach achieved $76.37 \%$ SEN \& $88.77 \%$ SPE (through EI configuration) compared to $71.02 \%$ SEN \& $88.53 \%$ SPE using the EEG features alone and 59.69\% SEN \& 69.21\% SPE using the ECG features only. They also reported that patient-independent approach accomplished 74.39\% SEN \& 66.95\% SPE (through EI configuration) compared to $68.18 \%$ SEN \& $73.95 \%$ SEP using the EEG features alone and 69.51\% SEN \& 62.22\% SPE using the ECG features only. The results were based on a dataset of 12 recordings from 10 neonates containing 633 seizures

Table 6 Execution times for classification using full EEGHRV feature and reduce feature sets

\begin{tabular}{lll}
\hline Algorithm & Feature fusion & $\begin{array}{l}\text { Classifier fusion: } \\
\text { linear classifier }\end{array}$ \\
& $\begin{array}{l}\text { (1-NN classifier) } \\
\text { Execution time (s) }\end{array}$ & $\begin{array}{l}\text { \& 1-NN classifier } \\
\text { Execution time (s) }\end{array}$ \\
\hline $\begin{array}{l}\text { Proposed classifiers + optimal } \\
\text { feature sets }\end{array}$ & 40.13 & 48.55 \\
\hline $\begin{array}{l}\text { Proposed classifiers + full } \\
\text { feature sets }\end{array}$ & 209.34 & 189.95 \\
\hline
\end{tabular}

with a mean duration of $3.86 \mathrm{~min}$ and 11 normal, healthy control neonates.

From these reported results, the combination of EEG and ECG algorithm in patient-specific scheme seem to outperform the patient-independent scheme. This observation is an agreement with previous works on automatic seizure detection using EEG [5,10-13]. However, what is usually desirable is to have a high performance seizure detector that is patient independent. Furthermore, the improvement achieved by the EEG and ECG combination method does not seem to be significant enough to warrant the use of a computationally demanding algorithm. Another point is that the patient-independent method did not improve over the EEG-based seizure detection but in fact has resulted in a lower specificity.

Our investigation led us to suggest two reasons for the limitation of the proposed schemes. First, the method in [38] uses a number of EEG and ECG features extracted from the time and frequency domains. This implicitly preassumes that the EEG and ECG are either stationary or at least locally stationary and, as a consequence, ignores potentially highly discriminating non-stationary features. Second, a number of features were extracted from the multi-channel EEG and then combined with the features obtained from ECG to detect seizures. Using a high dimensional feature vectors in automatic detections/classifications is known to negatively affect the performance; unless a feature selection process is performed prior to classification. This problem is due to the use of potentially performance-damaging irrelevant and/or redundant features. The obtained results related to the nature of the selected optimal features in "EEG features" section and those dealing with the effect of feature selection on the performance of the seizure detectors (Tables 3, 4, and 5) clearly support these two conclusions.

To further evaluate the performance of our proposed classifier fusion-based seizure detector (our best performing approach), we compared it with the newborn seizure detector proposed by Greene et al. on the patientindependent basis. We have extracted the six features from each EEG channel and six features from a single channel ECG as suggested in [38] and classified them into seizures and non-seizures using statistical classifiers in an EI configuration as reported in [38]. Table 7 shows the results of the detection methods using our database. 
Table 7 Performance comparison of seizure detectors based on newborn EEG and HRV inform

\begin{tabular}{|c|c|c|c|c|c|c|}
\hline \multirow[t]{2}{*}{ Algorithm } & \multicolumn{2}{|l|}{ EEG } & \multicolumn{2}{|l|}{ HRV } & \multicolumn{2}{|c|}{ Fusion of EEG and HRV } \\
\hline & SEN & SPE & SEN & SPE & SEN & SPE \\
\hline Proposed seizure detector & 80.9 & 86.5 & 85.7 & 84.6 & 95.2 & 94.3 \\
\hline Green's seizure detector & 71.5 & 65.6 & 59.6 & 67.5 & 72.8 & 60.3 \\
\hline
\end{tabular}

Table 7 demonstrates the superiority of our proposed newborn seizure detection algorithm. As discussed above, the good results of our algorithm can be attributed to a number of factors. Our method uses a feature selection algorithm which improved classification accuracy by excluding redundant and non-discriminative features. The optimal features resulted from the feature selection process of "EEG features" section were mostly those extracted from the TF domain. Thus, the proposed method efficiently uses the non-stationary information embedded in both EEG and HRV. The stationarity assumption of the newborn EEG and HRV seems to be a major factor restricting the performance of the techniques proposed by Greene et al. Time domain and frequency domain methods are by themselves not efficient enough to extract the rich information from nonstationary signals such as EEG and HRV.

\section{Limitations of the study and how to overcome them}

As this is one of the earliest attempts to implement biophysical signals fusion for seizure detection [7,37], the study described in this article has a number of limitations. Most of these limitations, however, are directly or indirectly related to the size of the database used. In this section, we discuss some of these limitations and how we plan to overcome them in the future.

The main limitation of the study is the size of the database used. For practical reasons, it is desirable to have an automated seizure detector that runs online and uses continuous EEG signals of hours or even days long. After all, the aim of the automation is to either assist the neurologist by highlighting the signal sections of interest or in applications that require immediate action, such as prediction [54], to completely replace him or her. To address this limitation, the authors plan to validate their methods on larger databases, including normal recordings, obtained from different data acquisition systems. This allows to address three points crucial for success of any automated detector, namely between patients variability, robustness to the different data acquisition systems, and the effect of different labeling techniques. For an online implementation, the widely used sliding method will be implemented [10]. A post-processing stage similar to the one proposed by different authors $[10,11,19,31]$ will be adopted to further enhance the performance of the seizure detector.
Another limitation of this study is related to the reporting of detected seizures in terms of segments/ epochs instead of seizure events and the use of sensitivity and specificity as the only measures of detection performance. Concerning the first point, the decision to adopt seizure epoch detection was mainly dictated by the size of the database. Applying an event-based method on database with a small number of seizures tends to give either over optimistic results, in the case of long seizures, or over pessimistic ones, in the case of short seizures. The issue related to the second point, namely the measures used to assess the detection performance, attracted much debate in the literature [55]. Although there is no standard for reporting detection performance, two complementary procedures are gaining wide acceptance. These methods are the event-based and the time-based detection methods $[19,56]$. Temko et al. [56] correctly concluded that the best way to report the performance of a seizure detector is to include a number of different, but complementary, measures. This approach will be adopted in our future works.

To assess the merit of a seizure detector, it is a common practice to compare its performance to some of the existing methods. As the aim of this study was mainly to assess the added value of the HRV information to the seizure detection, this issue was restricted to a small number of existing methods. One of our future plans is to conduct a performance analysis that will include a number of existing methods; especially those dealing with high dimensional feature spaces.

One of the most challenging tasks in automated seizure detection is dealing with artifacts contaminating the EEG. In this study, only those artifacts whose spectral energies lie outside the $(0.5-8 \mathrm{~Hz})$ frequency band were targeted. The fact that the performance did not suffer much may be explained by the small duration of the recordings used in this study. This, however, may not be the case for long-term continuous recordings. To deal with this situation, we plan to include some of the artifact removal techniques we previously developed [57$60]$ that deal with a wide variety of artifacts.

To detect newborn seizures, we have only considered a small number of statistical classifiers and few basic rules for classifier combination. These classifiers and combination rules were selected for their ease of implementation and computational efficiency. They have also been shown to perform well for a wide range of problems [53,61]. A 
number of more advanced methods [61-63] will be reviewed and adapted to the problem of seizure detection.

\section{Conclusions}

We proposed new approaches for detecting newborn seizure through the combination of information extracted from multi-channel EEG and single channel ECG. Two approaches for combining EEG and HRV were proposed in this article, namely feature fusion and classifier/decision fusion. The feature fusion was achieved by concatenating the features vectors extracted from the EEG and the HRV signals while the classifier fusion was accomplished by fusing the independent decisions from individual classifiers based on EEG and HRV. The combination of information from EEG and HRV has led to better performing automatic neonatal seizure detectors compared to detectors based solely on EEG $(+14.30 \%$ SEN,$+13.40 \%$ SPE $)$ or HRV $(+9.50 \%$ SEN, $+9.70 \%$ SPE). The decision-based fusion of EEG and HRV was generally found to give better specificity than the feature-based fusion (94.30\% versus. $88.60 \%)$. The results presented in this article confirm that information from physiological signals that directly reflect neurological changes (EEG) and signals that reflect autonomic behavior (HRV) complement each other and their fusion offers superior seizure detection performance.

\section{Competing interests}

The authors declare that they have no competing interests.

\section{Acknowledgments}

The authors would like to acknowledge Dr. Chris Burke and Ms. Jane Richmond from the Royal Children's Hospital, Brisbane, Australia, for their assistance in recording, labeling, and interpreting the data used in this study. This study was partly funded by an Australian Research Council Discovery grant (DP0665697) until 2009, and after that by QNRF under NPRP-09-465-2174

\section{Author details}

'School of Mechanical and Chemical Engineering, The University of Western Australia, Crawley, WA 6009, Australia. ${ }^{2}$ Faculty of Health Science and Biomedical Engineering, Universiti Teknologi Malaysia, 80990, Johor Bahru, Johor, Malaysia. ${ }^{3}$ University of Queensland Centre for Clinical Research, Building 71/918, Royal Brisbane \& Women's Hospital Campus, Herston, QLD 4029, Australia. ${ }^{4}$ College of Engineering, Qatar University, Doha, Qatar.

Received: 30 November 2011 Accepted: 4 September 2012 Published: 7 October 2012

\section{References}

1. JJ Volpe, Neonatal seizures, in Neurology of the Newborn, ed. by JJ Volpe, 4th edn. (WB Saunders, Philadelphia, 2000)

2. G Holmes, Childhood-specific epilepsies accompanied by developmental disabilities: causes and effects, in Epilepsy and Developmental Disabilities, ed. by O Devinsky, LE Westbrook (Butterworth-Heinemann, Melbourne, 2001)

3. W Deburchgraeve, PJ Cherian, M De Vos, RM Swarte, JH Blok, GH Visser, P Govaert, S Van Huffel, Automated neonatal seizure detection mimicking a human observer reading EEG. Clin. Neurophysiol. 119(11), 2447-2454 (2008)

4. J.J. Volpe, Neonatal seizures: current concepts and revised classification. Pediatrics 84, 422-428 (1989)

5. A Liu, JS Hahn, GP Heldt, RW Coen, Detection of neonatal seizures through computerized EEG analysis. Electroencephalogr. Clin. Neurophysiol. 82, 363369 (1992)
6. M Roessgen, B Boashash, A Comparison of Two Techniques for Detecting Seizure in Newborn EEG Data. Proceedings of the International Conference on Acoustics, Speech and Signal Processing 6, 3101-3104 (1996). Atlanta, Georgia, USA

7. B Boashash, M Keir, Design of a DSP System for Automatic Detection of Seizure Signals in Newborn. Proceedings of 5th International Symposium on Signal Processing and Its Applications, Brisbane, Australia 1, 351-354 (1999). Brisbane, Australia

8. B Boashash, AM Zoubir, M Roessgen, "On-Line Detection of Seizure in Newborn EEG Using Signal Processing Tools". Proceedings of the 13th International Conference on Digital Signal Processing 1, 79-82 (1997). Santorini, Greece

9. M Roessgen, B Boashash, Seizure analysis of newborn EEG using a model based approach. Proceedings of the International Conference on Acoustics, Speech and Signal Processing 1995 (ICASSP-95) 3, 1936-1939 (1995). Detroit, Michigan, USA

10. P Celka, PB Colditz, A computer-aided detection of EEG seizures in infants: a singular-spectrum approach and performance comparison. IEEE Trans. Biomed. Eng. 49, 455-462 (2002)

11. J Gotman, D Flanagan, J Zhang, B Rosenblatt, Automatic seizure detection in the newborn: methods and initial evaluation. Electroencephalogr. Clin. Neurophysiol. 103, 356-362 (1997)

12. B Boashash, M Mesbah, A time-frequency approach for newborn seizure detection. IEEE Eng. Med. Biol. Mag. 20(5), 54-64 (2001)

13. P Zarjam, M Mesbah, B Boashash, Detection of newborn EEG seizure using optimal features based on discrete wavelet transform. Proceedings of the International Conference on Acoustics, Speech and Signal Processing 2, 265-268 (2003). Hong Kong, China

14. H Hassanpour, M Mesbah, B Boashash, Time-frequency feature extraction of newborn EEG seizure using SVD-based techniques. EURASIP J. Appl. Signal Process. 16, 2544-2554 (2004)

15. PJ Cherian, W Deburchgraeve, RM Swarte, M De Vos, P Govaert, S Van Huffel, GH Visser, Validation of a new automated neonatal seizure detection system: a clinician's perspective. Clin. Neurophysiol. 122, 1490-1499 (2011)

16. S Faul, G Gregorcic, G Boylan, W Marnane, G Lightbody, S Connolly, Gaussian process modeling of EEG for the detection of neonatal seizures. IEEE Trans. Biomed. Eng. 54(12), 2151-2162 (2007)

17. M Roessgen, A Zoubir, B Boashash, Seizure detection of newborn EEG using a model based approach. IEEE Trans. Biomed. Eng. 45(6), 673-685 (1998)

18. J Altenburg, RJ Vermeulena, RLM Strijers, WPF Fetter, CJ Stam, Seizure detection in the neonatal EEG with synchronization likelihood. Clin. Neurophysiol. 114, 50-55 (2003)

19. MA Navakatikyan, PB Colditz, CJ Bruke, TE Inder, J Richmond, CE Williams, Seizure detection algorithm for neonates based on wave-sequence analysis. Clin. Neurophysiol. 117, 1190-1203 (2006)

20. J. Gotman, D. Flanagan, B. Rosenblatt, A. Bye, E. Mizrahi, Evaluation of an automatic seizure detection methods for the newborn EEG Electroencephalogr. Clin. Neurophysiol. 103, 363-369 (1997)

21. NB Karayiannis, A Mukherjee, JR Glover, PY Ktonas, JD Frost Jr., RA Hrachovy, EM Mizrahi, Detection of pseudosinusoidal epileptic seizure segments in the neonatal EEG by cascading a rule-based algorithm with a neural network. IEEE Trans. Biomed. Eng. 53(4), 633-641 (2006)

22. H Hassanpour, M Mesbah, B Boashash, Time-frequency based newborn EEG seizure detection using low and high frequency signatures. Physiol. Meas. 25, 934-944 (2004)

23. M Khlif, M Mesbah, B Boashash, PB Colditz, Multichannel-based newborn EEG seizure detection using time-frequency matched filter. Proceedings of the 29th Annual International Conference of the IEEE Engineering in Medicine and Biology Society, 1265-1268 (2007). Lyon, France

24. M Mesbah, M Khlif, B Boashash, PB Colditz, Newborn EEG seizure detection using optimized time-frequency matched filter. Proceedings of the International Symposium on Signal Processing and Its Applications, (CD ROM) (2007). Sharjah, UAE

25. L Rankine, M. Mesbah, B Boashash, A matching pursuit-based signal complexity measure for the analysis of newborn EEG. Med. Biol. Eng. Comput. 45(3), 251-260 (2007)

26. M Khlif, M Mesbah, B Boashash, PB Colditz, Detection of neonatal EEG seizure using multichannel matching pursuit. Proceedings of the 30th 
Annual International Conference of the IEEE Engineering in Medicine and Biology Society, 907-910 (2008). Vancouver, Canada

27. S. Faul, G. Boylan, S. Connolly, M. Liam, L. Gordon, An evaluation of automated neonatal seizure detection methods. Clin. Neurophysiol. 116, 1533-1541 (2005)

28. A Aarabi, F Wallois, R Grebe, Automated neonatal seizure detection: a multistage classification system through feature selection based on relevance and redundancy analysis. Clin. Neurophysiol. 117, 328-340 (2006)

29. A Aarabi, R Grebe, F Wallois, A multistage knowledge-based system for EEG seizure detection in newborn infants. Clin. Neurophysiol. $118,2781-2797$ (2007)

30. BR Greene, WP Marnane, G Lightbody, RB Reilly, GB Boylan, Classifier models and architectures for EEG-based neonatal seizure detection. Physiol. Meas. 29, 1157-1178 (2008)

31. A Temko, E Thomas, W Marnane, G Lightbody, G Boylan, EEG-based neonatal seizure detection with support vector machines. Clin. Neurophysiol. 122, 464-473 (2011)

32. MB Malarvili, M Mesbah, B Boashash, Time-frequency analysis of heart rate variability for neonatal seizure detection. EURASIP J Appl. Signal Process. (2007). doi:10.1155/2007/50396

33. MB Malarvili, M Mesbah, Newborn seizure detection based on heart rate variability. IEEE Trans. Biomed. Eng. 56(11), 2594-2603 (2009)

34. M Mesbah, MB Krishnan, P Colditz, B Boashash, Time-frequency based newborn seizure detection using heart rate variability, in Advanced Biosignal Processing, ed. by A. Nait-Ali (Springer-Verlag, Berlin, 2009)

35. BR Greene, P de Chazal, GB Boylan, S Connolly, RB Reilly, Electrocardiogram based neonatal seizure detection. IEEE Trans. Biomed. Eng. 54(4), 673-682 (2007)

36. OM Doyle, A Temko, W Marnane, G Lightbody, GB Boylan, Heart rate based automatic seizure detection in the newborn. Med. Eng. Phys. 32, 829-839 (2010)

37. B. Boashash, P. Barklem, M. Keir, Detection of seizure signals in newborns Proc ICASSP 4, 2351-2354 (1999)

38. BR Greene, GB Boylan, RB Reilly, P de Chazal, S Commolly, Combination of EEG and ECG for improved automatic neonatal seizure detection. Clin. Neurophysiol. 118, 1346-1359 (2007)

39. Task Force of the European Society of Cardiology and the North American Society of Pacing and Electrophysiology, Heart rate variability: standards of measurement, physiological interpretation, and clinical use. Eur. Heart J 17, 354-381 (1996)

40. MS Scher, M Sun, DA Steppe, RD Guthrie, RJ Sclabassi, Comparison of EEG spectral and correlation measures between healthy term and preterm infants. Pediatr. Neurol. 10(2), 104-108 (1994)

41. B Boashash (ed.), Time- Frequency Signal Analysis and Processing: A Comprehensive Reference (Elsevier, Oxford, UK, 2003)

42. L Rankine, M Mesbah, B Boashash, IF estimation for multicomponent signals using image processing techniques in the time-frequency domain. Signal Process. 87, 1234-1250 (2007)

43. B Boashash, Estimating and Interpreting the Instantaneous Frequency of a Signal-Part I: Fundamentals. Proceedings of the IEEE 80(4), 519-538 (1992)

44. B Boashash, Estimating and Interpreting the Instantaneous Frequency of a Signal-Part II: Algorithms. Proceedings of the IEEE 80(4), 539-569 (1992)

45. B Hjorth, The physical significance of time domain descriptors in EEG analysis. Electroenceph. Clin. Neurophysiol. 34, 321-325 (1973)

46. H Liu, H Motoda (eds.), Computational Methods of Feature Selection (Chapman \& Hall/CRC, Boca Raton, FL, 2008)

47. M Dash, H Liu, Feature selection for classification. Intell. Data Anal. 1, 131-156 (1997)

48. MB Malarvili, M Mesbah, B Boashash, HRV Feature selection based on discriminant and redundancy analysis for neonatal seizure detection. Proceedings of the 6th International Conference on Information, Communications \& Signal Processing, 1-5 (2007). Singapore

49. MB Malarvili, M Mesbah, B Boashash, HRV feature selection for neonatal seizure detection: a wrapper approach. Proceedings of the International Conference on Signal Processing and Communications, 864-867 (2007). Dubai, UAE

50. MB Malarvili, M Mesbah, B Boashash, Newborn seizure detection based on fusion of multi-channel EEG. Proceeding of the Workshop on Signal Processing and Its Applications, (CDROM) (2008). Sharjah, UAE

51. PA Devijver, I Kittler (eds.), Pattern Recognition: A Statistical Approach (Prentice-Hall, Englewood Cliffs, NJ, 1982)
52. J Kittler, M Hatef, R Duin, J Matas, On combining classifiers. IEEE Trans. Pattern Anal. Mach. Intell. 20(3), 226-239 (1998)

53. M Balakrishnan, Combining newborn EEG and HRV for automatic seizure detection. PhD Thesis, School of Medicine, the University of Queensland (2008)

54. B Litt, J Echauz, Prediction of epileptic seizures. Lancet Neurol. 1, 22-30 (2002)

55. S Vanhatalo, Development of neonatal seizure detectors: an elusive target and stretching measuring tapes. Clin. Neurophysiol. 122, 435-437 (2011)

56. A Temko, E. Thomas, W Marnane, G Lightbody, G Boylan, Performance assessment for EEG-based neonatal seizure detectors. Clin. Neurophysiol. 122, 474-482 (2011)

57. P Celka, B Boashash, P Colditz, Preprocessing and time-frequency analysis of newborn EEG seizures. IEEE Eng. Med. Biol. 2, 30-39 (2001)

58. P Zarjam, M Mesbah, Discrete wavelet transform based seizure detection in newborns EEG signals. Proceedings of 5th International Symposium on Signal Processing and Its Applications 2, 459-462 (2003). Paris, France

59. J Brotchie, LJ Rankine, M Mesbah, PB Colditz, B Boashash, Robust timefrequency analysis of newborn EEG seizure corrupted by impulsive artifacts. Proceedings of the 29th Annual International Conference of the IEEE Engineering in Medicine and Biology Society, 1265-1268 (2007). Lyon, France

60. MS Khlif, M Mesbah, B Boashash, P Colditz, Detection of neonatal seizure using multiple filters. Proceedings of the International Conference on Information Sciences, Signal Processing and their Applications, 284-287 (2010). Kuala Lumpur, Malaysia

61. S Theodoridis, K. Koutroumbas, Pattern Recognition, 4th edn. (Academic Press, Burlington, MA, 2009)

62. A Webb, KD Copsey, Statistical Pattern Recognition, 3rd edn. (John Wiley \& Sons, West Sussex, UK, 2011)

63. LI Kuncheva, Combining Pattern Classifiers: Methods and Algorithms (John Wiley \& Sons, New Jersey, 2004)

doi:10.1186/1687-6180-2012-215

Cite this article as: Mesbah et al: Automatic seizure detection based on the combination of newborn multi-channel EEG and HRV information. EURASIP Journal on Advances in Signal Processing 2012 2012:215.

\section{Submit your manuscript to a SpringerOpen ${ }^{\odot}$ journal and benefit from:}

- Convenient online submission

- Rigorous peer review

- Immediate publication on acceptance

- Open access: articles freely available online

- High visibility within the field

- Retaining the copyright to your article

Submit your next manuscript at $\gg$ springeropen.com 